\title{
Laser synthesized gold nanoparticles for high sensitive strain gauges
}

\author{
Salamat Burzhuev, Aykutlu Dâna, Bülend Ortaç* \\ UNAM-Institute of Materials Science E Nanotechnology, Bilkent University, Ankara 06800, Turkey
}

\section{A R T I C L E I N F O}

\section{Article history:}

Received 17 April 2013

Received in revised form 23 August 2013

Accepted 23 August 2013

Available online 7 September 2013

\section{Keywords:}

Laser ablation

Gold nanoparticles

Gold nanoparticle films

Quantum tunneling effect

Strain gauges

\begin{abstract}
A B S T R A C T
We demonstrate high strain sensitivity property of gold nanoparticle (Au-NP) thin films fabricated on flexible polydimethylsiloxane (PDMS) substrates. This behavior is attributed to quantum tunneling effect that is highly dependent on nanoparticle separation. Au-NPs were synthesized in water by nanosecond laser ablation method. The clean surface providing high tunneling decay constant, size of the Au-NPs and Au-NPs aggregate clusters offer advantages for high sensitivity strain sensor. We prepared Au-NPs films on flexible PDMS substrate by using hands-on drop-cast method. To obtain high gauge factor ( $g$ factor), we investigated the nanoparticles concentration on the substrate. Laser-generated Au-NPs films demonstrated $g$ factor of $\sim 300$ for higher than $0.22 \%$ strain and $\sim 80$ for the strain lower than $0.22 \%$ strain, which is favorably comparable to reported sensitivities for strain sensors based on Au-NPs. Mechanical characterizations for the prolonged working durations suggest long term stability of the strain sensors. We discuss several models describing conductance of films in low and high strain regimes.
\end{abstract}

(c) 2013 Elsevier B.V. All rights reserved.

\section{Introduction}

Strain gauges, which convert strain input to resistance change, find wide application in transduction of mechanical signals. Strain gauges based on metal and semiconductor films are commonly used in sensing. The sensitivity of a strain gauge is expressed by the $g$ factor, which can be calculated by the following equation:

$g=\frac{\Delta R}{R \varepsilon}$

where $\Delta R$ is the change in resistance upon applied strain; $R$ is the initial resistance; and $\varepsilon$ is the applied strain. The conventional metal film strain sensors have long lifetimes and are relatively robust to environmental effects such as changes in temperature and humidity. On the other hand, these sensors present low sensitivity due to the limited $g$ factors. Semiconductor based strain sensors have fundamentally different operation mechanism, namely modification of band structure, effective mass and mobility. Moreover, they are more sensitive, but may require temperature compensation. In addition, the sensitivity of these sensors decrease under high strains and a breakdown strain $\sim 0.6 \%$ resulting in limited operation range $[1,2]$.

Thin films based on nanomaterials have found wide applications in fields of optics, biology and detection. Metallic nanoparticles have gained significant attention mainly because of their unique optical, electrical and catalytic properties. In the literature,

\footnotetext{
* Corresponding author. Tel.: +90 3122903526.

E-mail address: ortac@unam.bilkent.edu.tr (B. Ortaç).
}

the conduction behavior between chemically synthesized nanoparticles has been intensively studied [3,4]. The conduction of $n$-alkanelthiol-stabilized Au-NP films was shown to have exponential decay dependence to the length of ligands' alkanethiolate chains. This effect was suggested to be attributed to quantum tunneling effect $[3,4]$. The quantum tunneling effect is highly dependent on width of the potential barrier. Thus, it was anticipated that the Au-NPs films can be used as highly-sensitive strain gauges. In principle, such films can be used in other types of sensors. For instance, one can measure the change conductance to observe the effect of chemical modification or attachment of biomolecules, which occurs due to changes in the tunneling barrier width and height.

The use of Au-NPs in strain sensing has previously been reported in literature. Herrmann et al. demonstrated the high capability of Au-NP films to sense small linear strains [5]. The Au-NP strain gauges were shown to be nearly two orders of magnitude sensitive than the conventional strain gauges [5]. Vossmeyer et al. demonstrated lower sensitivity Au-NP strain sensors fabricated on flexible substrates by depositing 12-dodecylamine-stabilized Au-NPs via layer by layer self-assembly on oxidized flexible polyethylene. This technique provides high adhesion of Au-NPs on the substrate because of linker compound, 1,9-nonanedithiol, which also increases the mechanical robustness of sensors [6]. Ressier et al. developed stop-and-go convective self-assembly method to fabricate few micrometer wires based on Au-NPs, and demonstrated that monolayer wires of Au-NPs have higher strain sensitivity - $g$ factor of 132 - than multilayer wires [7-9].

Presence of chemical stabilizers on Au-NP surfaces potentially decrease strain sensitivity. Thin films based on metal nanoparticles 
having clean surfaces were predicted to have improved $g$ factors [5]. Recently, Tanner et al. demonstrated that Platinum (Pt) nanoparticle coatings obtained by sputtering in vacuum present high $g$ factors (700). They also studied how surface density affects the sensitivity of such devices $[10,11]$. Nanoparticles free of chemical stabilizers can be obtained by laser ablation method [12,13]. Unique laser-matter interaction properties open the door to the generation of a variety of nanoparticles [14]. Compared with other nanoparticle synthesis methods, laser ablation, especially in liquids, is a versatile method for generating colloidal, highly pure, and surfactant-free nanoparticles. In this paper, we have studied the conduction properties of thin films of Au-NPs, prepared by nanosecond laser ablation in deionized water. Au-NP thin films were obtained by simply drop-casting Au-NP colloidal solution on the PDMS substrates.

\section{Experimental details}

\subsection{Au-NP synthesis by laser ablation in liquid}

Au-NPs were obtained by laser ablation in deionized water. Gold block (99.999\%, Kurt J. Lesker) was cleaned by sonification in acetone prior to laser ablation without any additional purification. The generation of colloidal nanoparticles from Au block was carried out by using a commercial pulsed ND:YLF laser (wavelength: $\lambda=527 \mathrm{~nm}, 16 \mathrm{~W}$ average power, pulse duration $t=100 \mathrm{~ns}$, pulse energy $E=16 \mathrm{~mJ}$ for $1 \mathrm{kHz}$ ). The cleaned Au block was placed in a glass vessel containing $23.5 \mathrm{~mL}$ of pure deionized water. The laser beam was focused on the target by using a plano-convex lens with focal length of $50 \mathrm{~mm}$. The height of liquid layer over the gold target is $\sim 5 \mathrm{~mm}$. The laser ablation was carried out for $\sim 10 \mathrm{~min}$, and the laser beam is scanned over the target surface. During the laser ablation, the formation of colloidal nanoparticle solution with dispersed Au-NPs in liquid media was observed as a color change of the deionized water. After the laser irradiation, the color of the Au-NPs solution became dark-red.

The structure of Au-NPs generated by nanosecond pulsed laser ablation were studied by TEM (TEM model FEI - Tecnai G2F30) system. Sample for TEM analysis was prepared by drop-casting solutions onto carbon-coated grid. Representative TEM image of the Au-NPs is shown in Fig. 1, showing well dispersed, sphericallike NPs. Size distribution was measured from TEM image where 100 particles' approximate diameters were measured. Size distribution of Au-NPs is given in Fig. 1 inset. An average nanoparticles size of $13 \mathrm{~nm}$ is seen, with a distribution covering the range from 2 to $25 \mathrm{~nm}$.

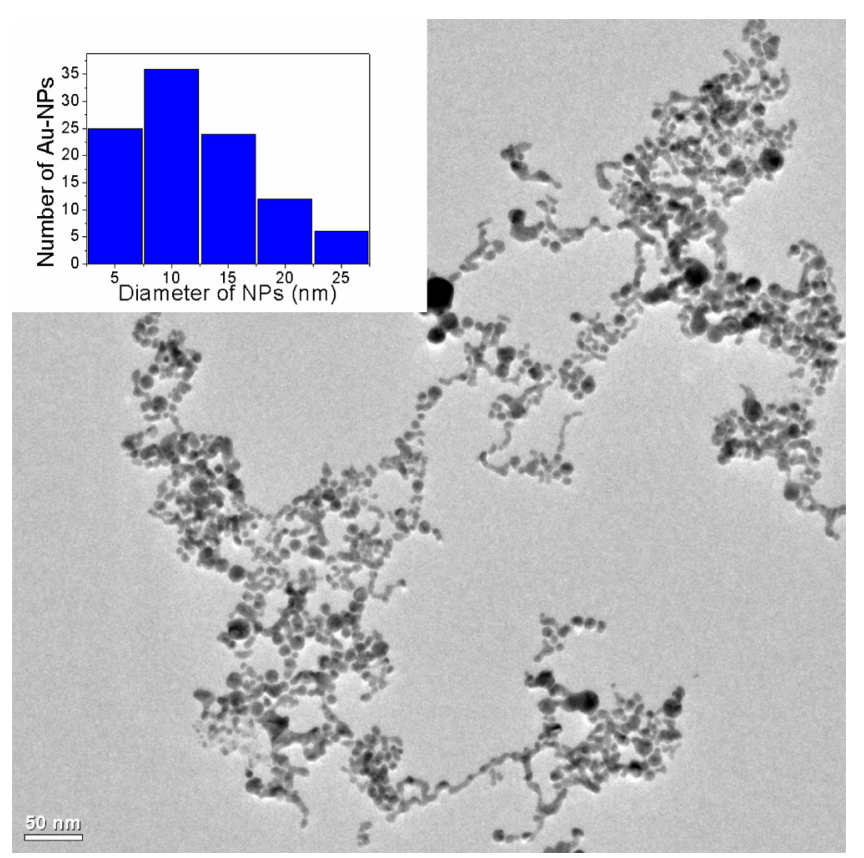

Fig. 1. TEM image of Au-NPs. The inset shows the histogram of size distribution calculated from TEM images.

\subsection{Strain sensor fabrication}

Thin films of Au-NPs have been produced by drop-casting on several substrates. It was observed that, uniformity of the films were poor on $\mathrm{SiO}_{2}$ and silicon substrates due to their hydrophilic surfaces. Au-NPs on glass and silicon were randomly distributed and intensively located at the edge of the initial Au-NP droplet's contact area. On the other hand, PDMS substrate presents low surface energy, thus a hydrophobic surface. The high contact angle and low surface energy enables to uniformly precipitate particles and highly concentrate them on the surface. After droplet was completely dried, a dark blue spot was formed on the surface of PDMS. Au-NP thin films obtained on PDMS were uniformly distributed (Fig. 2a). Contacts were obtained using a shadow mask and a $350 \mu \mathrm{m}$ gap was formed by coating $80 \mathrm{~nm}$ Pt film using sputtering (PECS Gatan 682) (Fig. 2b).

Scanning electron microscope (SEM) (FEI Nova NanoSEM 600) was used to further investigate the localization of Au-NPs on the PDMS substrate. In the SEM image, aggregated clusters can be

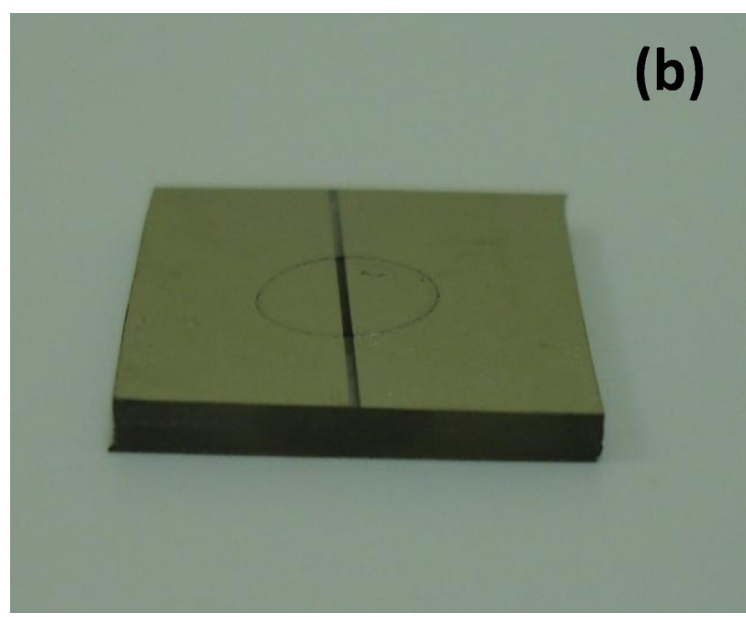

Fig. 2. Au-NPs film on PDMS substrate. (a) Au-NPs film after droplet was dried. (b) After Pt contacts were deposited by PECS. 


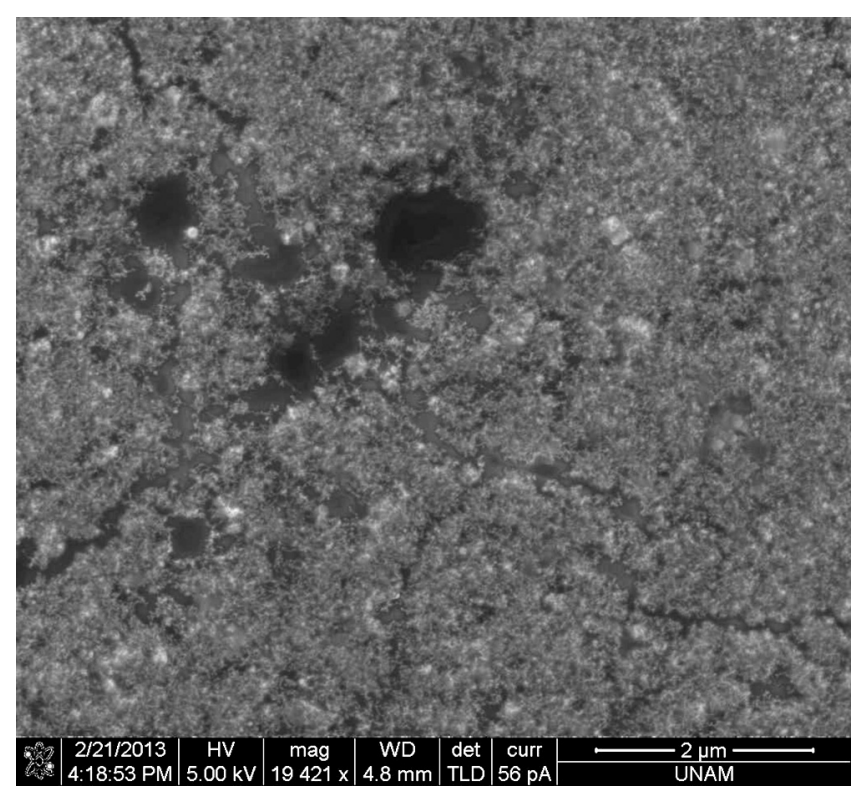

Fig. 3. SEM image of Au-NPs thin film on PDMS substrate.

realized, through which conduction takes place (Fig. 3). Also, PDMS surface is not fully covered by Au-NPs and there are cracks (Fig. 3). These may increase resistance of Au-NPs film.

\subsection{Mechanical characterization}

Au-NP film on the PDMS substrate was characterized by applying linear strain and observing change in resistance as shown in Fig. 4. In this figure, Au-NP strain sensor is schematically shown, where Au-NPs and aggregates of Au-NPs are move apart while tensile strain is applied; resulting in the change of resistance (Fig. 4). Aggregation of laser generated Au-NPs can be observed in Fig. 3. A linear translation stage fitted with a piezoelectric actuator (Thorlabs AE0203D08F) was used to characterize the strain response of Au-NPs films. In static measurements, using a fixed bias of $1 \mathrm{~V}$ current through the films was monitored using a precision current meter (Keithley 4200-SCS). Dynamic strain response of the AuNPs thin film strain sensor was monitored using a transimpedance amplifier (Stanford Research systems SR-570) and digital oscilloscope (Tektronix TDS1012B), Piezoelectric actuator was driven with a square function of frequency $1 \mathrm{~Hz}$ and peak-to-peak amplitude of $5 \mu \mathrm{m}$. Dynamic measurements were performed under $0.25 \mathrm{~V}$ bias applied to the film.

\section{Results and discussion}

The concentration of the nanoparticles on the substrate strongly affects the conductivity and strain sensitivity of the Au-NP film. The concentration of gold in the Au-NPs in water obtained by $\sim 10 \mathrm{~min}$ laser ablation was approximately $1.08 \mathrm{mM}$. Gold mass in Au-NP colloidal solution was calculated by weighing the gold block before and after the ablation process. Then the molarity value of gold in Au-NP solution was calculated in terms of mM. This colloidal solution was diluted several times to obtain solutions with different concentrations. $300 \mu$ l of diluted solutions were applied to PDMS substrates and dried at ambient conditions to form spots with typical diameter of $5 \mathrm{~mm}$. Films prepared with $>0.9 \mathrm{~mm}$ colloidal solutions were very conductive and did not respond to strain; while, films prepared with $<0.72 \mathrm{mM}$ solutions were highly resistive (resistance $>200 \mathrm{M} \Omega$ ) and sensitive to strain. It was observed that films that have higher resistance were more sensitive to strain. Optimal concentration for Au-NPs strain gauges is found to be about $\sim 0.81 \mathrm{mM}$ that yields high sensitivity and measurable resistance. Laser-synthesized nanoparticle solutions tended to aggregate due to absence of chemical stabilizers resulting in different film formation after several days.

In Fig. 5, it is clearly seen that there is significant change in resistance of Au-NP film while the strain is being applied, which

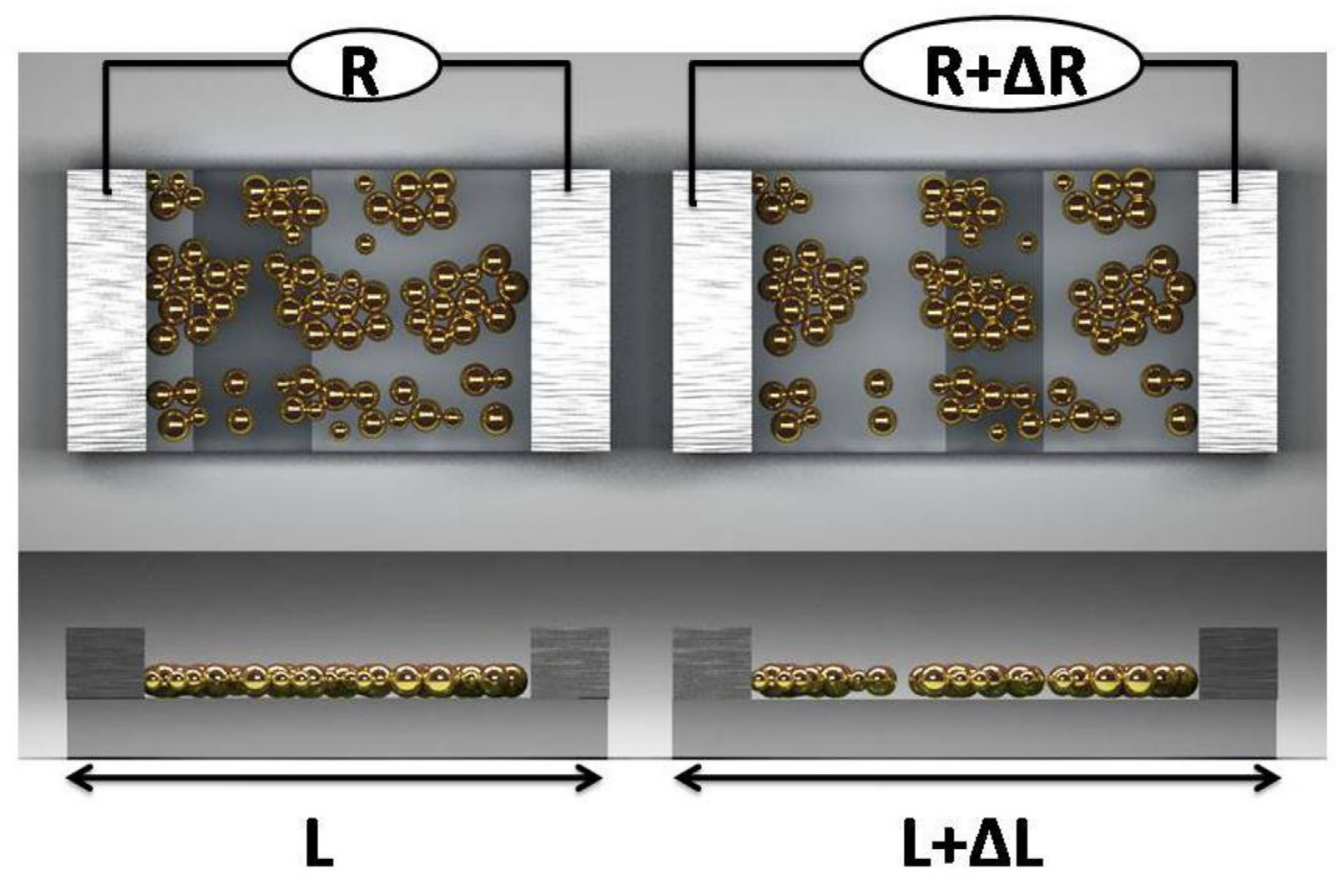

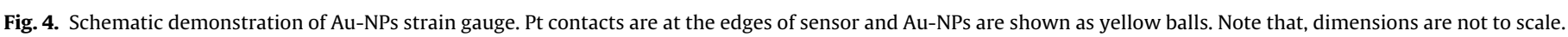

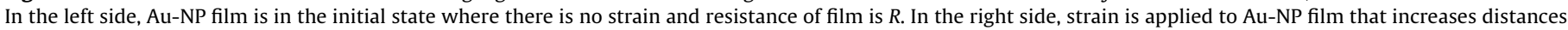

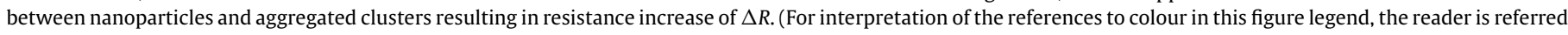
to the web version of this article.) 


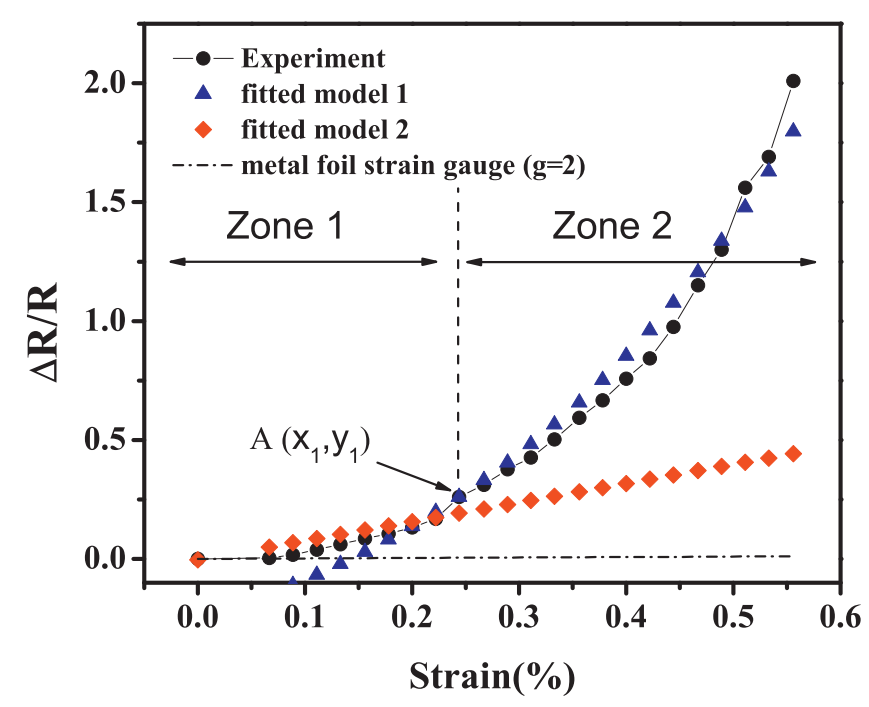

Fig. 5. Resistance change response of Au-NP films while applying strain. Fit 1 and 2 are the corresponding linear and exponential functions. Also, metal foil strain gauge response is shown for comparison which have $g$ factor of 2 (dashed-dotted line).

indicates higher sensitivity of Au-NP film than conventional metal foil strain gauges. Previously, the strain response of Au-NP strain sensor is explained by an exponential dependence as:

$\Delta R / R=\exp (g \varepsilon)-1$

where particles initially separated from each other [5]. Strain response of the Au-NP film with initial resistance of $20.8 \mathrm{M} \Omega$ is shown in Fig. 6. It is observed that the Au-NP strain sensor response is linear under small strains $(<0.22 \%)$. The $g$ factor is obtained by fitting a function of the form:

$\Delta R / R \approx g \varepsilon$

which results in $g \cong 80.2$ under small strains (Fig. 5, fit 1 ). Under higher strains $(>0.22 \%$ ) Au-NP film resistance is fitted with an exponential function of the form:

$\Delta R / R-y_{1}=\exp \left(g\left(\varepsilon-x_{1}\right)\right)-1$

where $\left(x_{1} y_{1}\right)$ is A point in Fig. 5 which results in an effective $g$ factor of $g \cong 298$ (Fig. 5, fit 2). $G$ factor is described by following equation:

$g=\beta(d+l)$

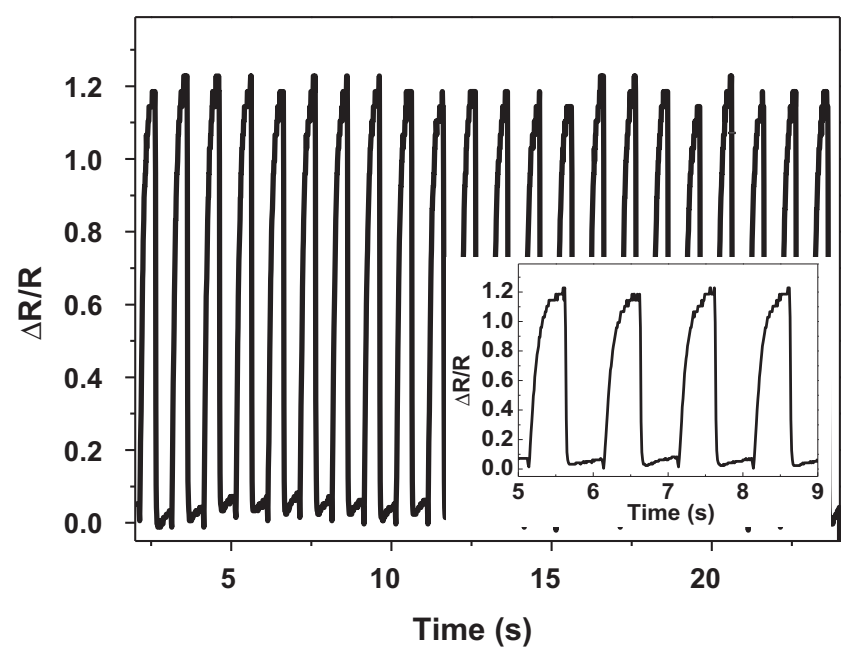

Fig. 6. Alternating strain response of Au-NPs strain sensor (at $1 \mathrm{~Hz}$ frequency). where $g$ is the $g$ factor; $d$ is the diameter of nanoparticle or aggregate of nanoparticles; $l$ is the distance between nanoparticles; $\beta$ is the tunneling decay constant [5]. Tunneling decay constant depends on the height of quantum barrier. Under low biases, $\beta=2 \sqrt{2 m \varphi / \hbar^{2}}$ where $m, \varphi$ and $\hbar$ are electron effective mass, work function and reduced Planck's constant, respectively.

Assuming that tunneling undergoes in air, tunneling decay constant is $\sim 20 \mathrm{~nm}^{-1}$ and average nanoparticle diameter is $\sim 13-15 \mathrm{~nm}$; so, $g$ can be approximated as a value of 300 . Aggregated clusters might be also responsible for high sensitivity of Au-NP strain gauge since every cluster can be considered as a particle. In this scenario, aggregates remain unstrained while most of the deformation occurs between them. As a result, Eq. (5) can be modified in such way that $d$ corresponds to average cluster diameter $[5,11]$.

One possible explanation for the presence of multiple regimes with distinct effective $g$ factors is sought by considering the conduction mechanism in the Au-NP film. Initially, by proper choice of NP concentration, the films are prepared near the percolation threshold, with a large number of parallel conduction paths. Under the application of a small strain (zone 1 in Fig. 5), the number of paths contributing to conductance of the films diminishes, causing a linear increase in resistance described by Eq. (3). As the paths are broken at point $A$, conduction through the paths are dominated by tunneling (zone 2 in Fig. 5), which exhibits a larger resistance change. At the point $A\left(x_{1}, y_{1}\right)$ quantum tunneling starts to play major role: as a result, Eq. (2) should be corrected to Eq. (4) so that exponential dependence begins from the point $\left(x_{1}, y_{1}\right)$.

Modulation strain response of Au-NP strain gauge shows dynamic response of resistance (Fig. 6). The initial resistance of this strain sensor used for this experiment was $11.11 \mathrm{M} \Omega$. G factor of Au-NP sensor used in this experiment is about 118. Strain response did not exhibit noticeable change after hours of operation. Also, reproducible responses can be taken even after weeks of initial preparation. For the frequencies larger than $1 \mathrm{~Hz}$, sensitivity of the device starts to degrade because of low Young's modulus and viscoelastic property of PDMS.

Resistance of Au-NP film is highly dependent on gaps between nanoparticles. Statistical study is required in order to understand how Au-NP film resistance is dependent on gaps between Au-NPs. We made simulation where every gap is considered to be either series or parallel resistor (Fig. 7). We perform numerical MonteCarlo simulations to understand how the gaps between Au-NPs affect the resistance of the Au-NP strain sensor. Simmons's generalized formula for tunneling was used to obtain resistances of the samples [15]. Potential difference between the nanoparticles is assumed as the distance between nanoparticles times electric field $(V=E l)$ where electric field equals to applied bias divided by distance between to contacts $\left(E=V_{\text {bias }} / L\right)$. Direct tunneling should be observed since potential difference between nanoparticles is small due to small ( $<1 \mathrm{~nm})$ distances between nanoparticles. In the simulation, input was a random Gaussian distribution of the gaps (700*10,000 2D nanoparticles matrix, performed 1000 times) and output was number of samples vs. resistance of the samples. Fig. 7 is seems as a Gaussian distribution, which shows the number vs. resistances of samples. The gap between nanoparticles is $0.18 \mathrm{~nm}$ with standard deviation of $0.18 \mathrm{~nm}$. In other words, the nanoparticles are very close to each other. This graph projects that different substrates appear while drying same amount of Au-NPs on PDMS.

Furthermore, laser synthesized Au-NP films present promising properties for sensing applications because these sensors are easily fabricated on PDMS substrate and have high strain sensitivity. PDMS, having low Young's modulus, is flexible substrate which can be advantageous for some applications such as low pressure sensing. It is easy to obtain uniform films on PDMS; however, this 

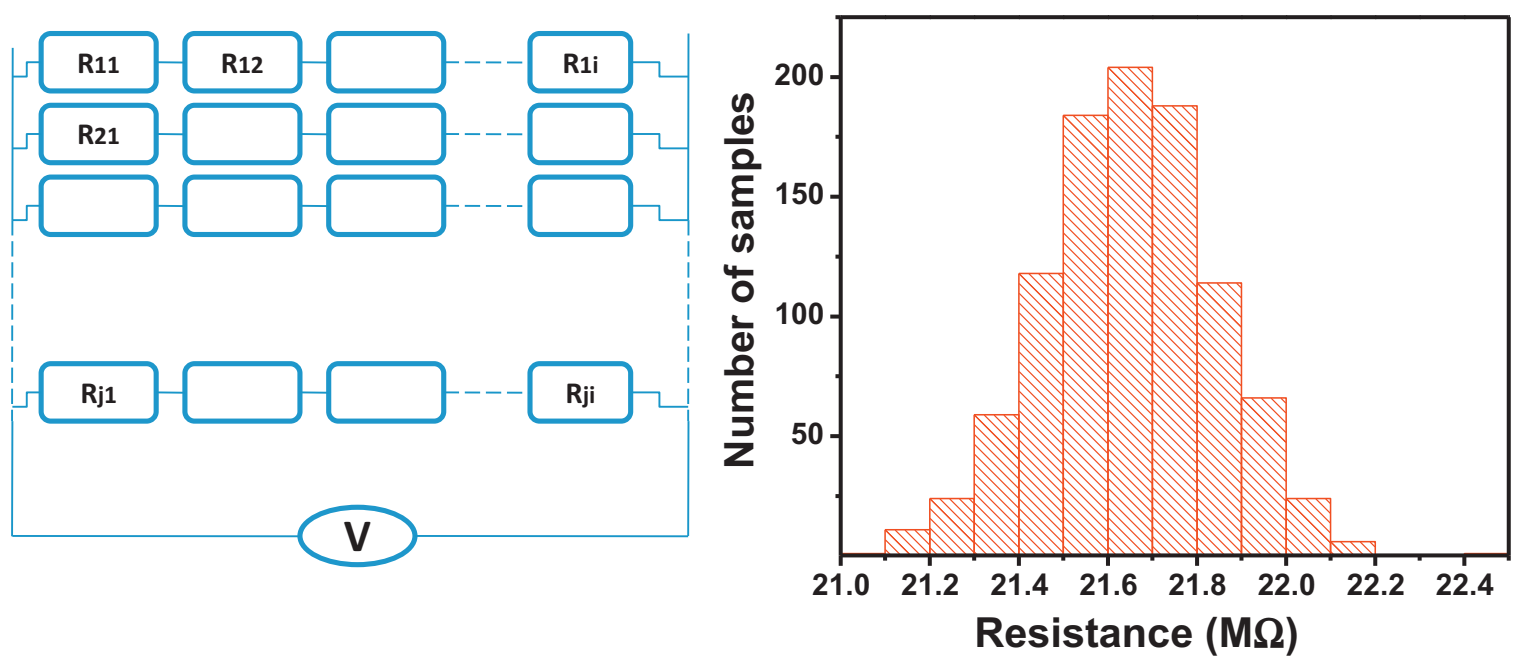

Fig. 7. Simple circuit model of Au-NPs thin film and histogram of number of samples vs. resistance of the samples.

substrate has some drawbacks such as creep deformation, stress relaxation and high thermal coefficient. Depending on sensing applications, different substrates with hydrophobic surface, which allows uniform deposition of nanoparticles by drop-cast method, can be employed to develop Au-NP strain gauge. For example, substrates with high Young's modulus can increase operation frequency range of the device. Studies on deposition techniques may be required to improve adhesion of Au-NPs on the substrate which may improve mechanical robustness. Coating Au-NP film with protection layer could be also needed to reduce unwanted environmental effects such as variations in humidity. Smaller contacts can be employed for decreasing nominal resistance which in turn will reduce noise associated with low current detection. In order to reduce variations in gauge factor, drop-casting on compressively strained substrate can be used. After relieving substrate it expands and all conducting path will be broken; as a result, only one regime (tunneling) will be responsible for resistance change in Au-NPs strain gauges. This will also make Au-NP film sensitive to compressive strain. For further studies; the reliability, the repeatability and the environmental effects such as gauge hysteresis, temperature dependence, long term aging, compression vs. tension deviation, sensitivity shift with number of cycles, etc. could be investigated for real-world applications of the Au-NP stain gauge.

\section{Conclusion}

In conclusion, we have demonstrated that laser generated AuNP films on PDMS can be further used for highly sensitive strain gauge. $G$ factor of these films is found to be $\sim 300$ for strains higher than $0.22 \%$, which is highest reported sensitivity for Au-NPs strain sensors. High sensitivity properties of laser generated Au-NP strain sensors was attributed to clean surfaces, size and aggregated clusters of Au-NPs. High stability was also studied by self-made mechanical characterizations. For the further investigation, the AuNP films yield interesting electrical properties that may be used in transition voltage spectroscopy.

\section{Acknowledgments}

State Planning Organization (DPT) of Turkey is acknowledged for the support of UNAM-Institute of Materials Science and Nanotechnology. Dr. Ortaç acknowledges the 'Industrial Thesis Projects Programme' of the Ministry of Industry and Trade for funding the San-Tez (636.STZ.2010-1) project.

\section{References}

[1] J.S. Wilson, Sensor Technology Handbook, Elsevier, Amsterdam, Boston, 2005.

[2] R.L. Hannah, S.E. Reed, Society for Experimental Mechanics (U.S.), Strain Gage Users' Handbook, Elsevier Applied Science/Society for Experimental Mechanics, London/New York/Bethel, CT, USA, 1992.

[3] F.P. Zamborini, M.C. Leopold, J.F. Hicks, P.J. Kulesza, M.A. Malik, R.W. Murray, Electron hopping conductivity and vapor sensing properties of flexible network polymer films of metal nanoparticles, Journal of the American Chemical Society 124 (2002) 8958-8964.

[4] W.P. Wuelfing, S.J. Green, J.J. Pietron, D.E. Cliffel, R.W. Murray, Electronic conductivity of solid-state, mixed-valent, monolayer-protected Au clusters, Journal of the American Chemical Society 122 (2000) 11465-11472.

[5] J. Herrmann, K.H. Muller, T. Reda, G.R. Baxter, B. Raguse, G.J.J.B. de Groot, et al., Nanoparticle films as sensitive strain gauges, Applied Physics Letters 91 (2007).

[6] T. Vossmeyer, C. Stolte, M. Ijeh, A. Kornowski, H. Weller, Networked goldnanoparticle coatings on polyethylene: charge transport and strain sensitivity, Advanced Functional Materials 18 (2008) 1611-1616.

[7] C. Farcau, N.M. Sangeetha, H. Moreira, B. Viallet, J. Grisolia, D. CiuculescuPradines, et al., High-sensitivity strain gauge based on a single wire of gold nanoparticles fabricated by stop-and-go convective self-assembly, ACS Nano 5 (2011) 7137-7143

[8] C. Farcau, H. Moreira, B. Viallet, J. Grisolia, L. Ressier, Tunable conductive nanoparticle wire arrays fabricated by convective self-assembly on nonpatterned substrates, ACS Nano 4 (2010) 7275-7282.

[9] C. Farcau, H. Moreira, B. Viallet, J. Grisolia, D. Ciuculescu-Pradines, C. Amiens, et al., Monolayered wires of gold colloidal nanoparticles for highsensitivity strain sensing, Journal of Physical Chemistry C 115 (2011) 14494-14499.

[10] J.L. Tanner, D. Mousadakos, P. Broutas, S. Chatzandroulis, Y.S. Raptis, D. Tsoukalas, Nanoparticle strain sensor, Procedia Engineer 25 (2011).

[11] J.L. Tanner, D. Mousadakos, K. Giannakopoulos, E. Skotadis, D. Tsoukalas, High strain sensitivity controlled by the surface density of platinum nanoparticles, Nanotechnology 23 (2012)

[12] J.P. Sylvestre, S. Poulin, A.V. Kabashin, E. Sacher, M. Meunier, J.H.T. Luong, Surface chemistry of gold nanoparticles produced by laser ablation in aqueous media, Journal of Physical Chemistry B 108 (2004) 16864-16869.

[13] H. Muto, K. Yamada, K. Miyajima, F. Mafune, Estimation of surface oxide on surfactant-free gold nanoparticles laser-ablated in water, Journal of Physical Chemistry C 111 (2007) 17221-17226.

[14] A.V. Simakin, V.V. Voronov, N.A. Kirichenko, G.A. Shafeev, Nanoparticles produced by laser ablation of solids in liquid environment, Applied Physics A 79 (2004) 1127-1132.

[15] J.G. Simmons, Generalized formula for the electric tunnel effect between similar electrodes separated by a thin insulating film, Journal of Applied Physics 34 (1963) 1793-1803.

\section{Biographies}

Salamat Burzhuev is currently a M.S. student at Institute of Materials Science and Nanotechnology, Bilkent University, Ankara, Turkey. He received the B.S. in Physics from Middle East Technical University, Ankara, Turkey in 2006. His current research interests include ultrafast lasers and its applications; nanomaterials for sensor development. 
Dr. Aykutlu Dâna received his B.S. degree in Electrical Engineering from Bilkent University, Ankara, Turkey in 1995, M.S. degree Electrical Engineering from Stanford University, CA, USA in 1998, and Ph.D. degree in Electrical Engineering from the Stanford University in 2003. He is currently working as a research assistant professor at Institute of Materials Science and Nanotechnology, Bilkent University. His current research areas cover fundamental and applied studies of micro and nanoscale opto-electronic devices and systems; surface plasmon resonance based biosensors: micromachined surgical tools; modelling, design, fabrication and characterization of semiconductor devices with novel nanostructured features; X-Ray photoelectron spectroscopy for electrical characterization; nanomechanics, force microscopy and spectroscopy.
Dr. Bülend Ortac received the B.S. degree in Physics from the Karadeniz Technical University, Trabzon, Turkey, in 1997, M.S. degree in Teaching and Diffusion of Sciences and Technology from ENS Cachan University, Paris, France, in 2000, and $\mathrm{Ph}$. D. degree in Optoelectronics from Rouen University, Rouen, France, in 2004 respectively. In Mars 2005, he joined the Institute of Applied Physics, FriedrichSchiller University, Jena, Germany, as a Post-Doctoral Associate. Since November 2009, he has been working as a research assistant professor at Institute of Materials Science and Nanotechnology, Bilkent University. His current research interests include the development of powerful fiber lasers in the continuous-wave regime to pulsed regime (ns, ps and fs) and the demonstration of laser systems for real world applications. 